product, actinium $\mathrm{X}$, could be separated from actinium. Actinium $X$ produces the emanation, and this in turn the active deposit. The similarity between these two substances is even closer, for I have found that a new product is present in actinium which is intermediate between actinium and actinium $\mathrm{X}$, and, from analogy to thorium, will be called for convenience " radio-actinium." This product emits $\alpha$ rays, is half-transformed in about twenty days, and is the parent of actinium X.

The separation of radio-actinium from an actinium solution in radio-active equilibrium can be of ten accomplished by producing a very small precipitate in the solution, which settles down slowly and carries with it the new product, while most of the actinium and actinium $X$ remain in the solution. Amorphous sulphur was found to be very convenient for that purpose. To a fairly strong hydrochloric acid solution of actinium some sodium thiosulphate was added, and the small amount of sulphur was allowed to settle down in the cold. After filtration the precipitate was tested for activity. It showed a strong $\alpha$-ray activity, but comparatively very little $\beta$-ray activity, and gave out very little emanation.

The $\alpha$-ray activity steadily rises to a maximum after about three weeks, the activity then being about two or three times its initial value. The activity then decays and ultimately according to an exponential law, with a period of about twenty days. The $\beta$-ray activity and emanating power reach a maximum at the same time, and decay with the same period. This rise of activity to a maximum is due to the formation of actinium $X$ from the radioactinium. This is shown by the increase of the emanating power, and was also verified by direct separation of actinium $\mathrm{X}$ from the radio-actinium. For instance, if one separates the actinium $\mathrm{X}$ when the activity of the radioactinium is decreasing, the activity of the residue again rises and varies in the same way as in the above described experiment. Actinium itself, freed from all its products, gives out practically no $\alpha$ or $\beta$ rays, but then slowly increases in activity, reaching a maximum after about four months. Godlewski obtained almost inactive actinium, showing that he had unknowingly separated the new product from the actinium. I have observed that when dissolving actinium in hydrochloric acid, generally a small portion remains undissolved, and this fraction contains radio-actinium to a large extent.

Giesel long ago stated that his preparations of emanium increased in activity for about six months. This may probably be explained by the formation of radio-actinium. In a recent paper, Marckwald (Ber. d. d. chem. G., I905, 2264) compared the chemical properties of actinium and emanium, and concluded that actinium and emanium were not identical, but the latter was the parent of the former. the activity of his actinium decaying in the course of several months. This is in contradiction with Debierne's statement that his actinium does not lose its activity. The decaying substance, separated by Marckwald, which he concluded to be the actinium of Debierne, may possibly be the product radio-actinium, because a precipitation of thorium with sodium thiosulphate carries down the radio-actinium also. But it remains to be explained why his actinium did not rise at first, or why it did not seem to contain actinium $\mathrm{X}$.

It may be mentioned that the above described experiments were carried out both with the actinium of Debierne and the emanium of Giesel. The same results were obtained in both cases.

A more complete account of these experiments will be given later.

McGill University, Montreal, March 27.

\section{The April Meteors.}

THESE meteors will return this year at a favourable period, the moon being near new and only visible as a slender crescent for a short time before sunrise. If the atmosphere should prove clear during the night following Saturday, April 2 I, a number of Lyrids will probably be seen. The shower is likely to reach its best after midnight, when the radiant at $27 \mathrm{I}^{\circ}+33^{\circ}$ will have attained a sufficiently high altitude to favour the visible distribution of its meteors.
The display is seldom very rich, and has not developed striking brilliancy since ${ }_{1} \mathrm{So}_{3}$, but it sometimes offers fairly conspicuous features, as in 1901 . Usually it does not equal the strength of the annual Perseid shower of August, and it is certainly of much shorter duration, for its period of special activity is often confined to a few hours. In 190 the Lyrids were pretty bright and plentiful on April 2I, though on the previous night the display could be scarcely recognised during a long watch. An interesting feature of this system is that its radiant, like that of the August meteors, exhibits a daily motion eastwards amongst the stars. This displacement is, however, very difficult to trace owing to the brief duration of the shower, and to its comparative feebleness at many of its returns.

Observers would do well to watch attentively for early Lyrias on April 18 and 19 , and for late members of the stream on April 22, 23, and 24 next. Individual meteors, accurately recorded on these dates, may be regarded as very valuable, since it will be possible to compare their paths with duplicate observations secured elsewhere, and thus their radiant points and heights in the air may be determined with trustworthy precision.

Meteors from streams contemporary with the Lyrids are usually somewhat rare, but in recent years two showers of slow-moving meteors have been well pronounced from southern positions at $189^{\circ}-31^{\circ}$ and $218^{\circ}-31^{\circ}$.

\section{W. F. Denning.}

THE interesting Lyrid meteor shower passed unobserved last year owing to the generally unsatisfactory state of the weather that prevailed at the time of its expected appearance. In the event of better atmospheric conditions obtaining at the present epoch, the Lyrids are likely to be strongly in evidence, as the circumstances that regulate the intensity of these meteor apparitions will be exceptionally favourable. According to calculations by the writer, the Lyrid shower will fall in 1906 on the night of April I9, and will be visible at least in part from both sides of the Atlantic, though the main bulk of the display will descend over the American continent. The earlier maxima on April ig fall due about roh. $30 \mathrm{~m}$. and r $4 \mathrm{~h}$. $3 \mathrm{om}$. G.M.T.; the second and stronger phase of the shower will culminate at Igh. $3 \mathrm{om}$, and will be followed by two other maxima, one of which occurs on April 19 23h. and the other on April 20 2h. The last and final outburst of meteoric activity will, of course, completely elude observations over the American continent. Of the minor showers associated with the period, the most prominent will be visible on the nights of April 23 and 25 ; on the former there is a well defined maximum at $13 \mathrm{~h}$., while on April 2,5 two or three maxima will take effect between oh. and I2h. $30 \mathrm{~m}$.

April 7 .

JOHN R. HENRY.

\section{Sea-sickness and Equilibration of the Eves.}

$I_{N}$ connection with the above subject, which Mr. Sang brought under the notice of your readers in your number for March 29, I would like to point out that it has been long known that the eves mav play an important part in sea-sickness. When making some investigations connected with a "New Variety of Ocular Spectrum" (Proc. Roy. Soc. Edin., vol. x.), I found that by acting on the eves alone a very disagreeable sickness, similar to sea-sickness, could be easily produced. The subject was comfortably seated in a chair with his head in a large cylindrical box. The box was open below, but partly closed on the top by a circular piece of wood by which the box was suspended. The cylindrical sides of the box were made of tracing paper having broad black vertical bands painted on it. When the box was rotated on a vertical axis, the black and white vertical bars passed in succession in front of the observer. The effects on the subjects were various: sometimes they felt as if they were rotating in a direction the opposite of that of the box, but the most certain resuit was a very disagreeable sickness, which continued for some time after the experiment was made. Personally, I find the best preventive of sea-sickness is to lie down and read anything I may be interested in, holding the book in such a position that it shuts out the view of all other objects.

Ardenlea, Falkirk, N.B., March $3 \bar{i}$.
John Aitkes.

No. I9O2, vol. 73] 